\title{
Human-Robot Interaction in Handing-Over Tasks
}

\author{
Markus Huber ${ }^{1}$, Markus Rickert ${ }^{2}$, Alois Knoll ${ }^{2}$, Thomas Brandt ${ }^{3}$, Stefan Glasauer ${ }^{1}$ \\ ${ }^{1}$ Center for Sensorimotor Research, Department of Neurology, Ludwig-Maximilians-Universität München \\ ${ }^{2}$ Robotics and Embedded Systems Lab, Department of Computer Science, Technische Universität München \\ ${ }^{3}$ Department of Neurology, Ludwig-Maximilians-Universität München
}

\begin{abstract}
In many future joint-action scenarios, humans and robots will have to interact physically in order to successfully cooperate. Ideally, seamless human-robot interaction should not require training for the human, but should be intuitively simple. Nonetheless, seamless interaction and cooperation involve some degree of learning and adaptation. Here, we report on a simple case of physical human-robot interaction, a hand-over task. Even such a basic task as manually handing over an object from one agent to another requires that both partners agree upon certain basic prerequisites and boundary conditions. While some of them are negotiated explicitly, e.g. by verbal communication, others are determined indirectly and adaptively in the course of the cooperation. In the present study, we compared human-human hand-over interaction with the same task done by a robot and a human. To evaluate the importance of biological motion, the robot human interaction was tested with two different velocity profiles: a conventional trapezoidal velocity profile in joint coordinates and a minimum-jerk profile of the end-effector. Our results show a significantly shorter reaction time for minimum jerk profiles, which decreased over the first three hand-overs. The results of our comparison provide the background for implementing effective joint-action strategies in humanoid robot systems.
\end{abstract}

\section{INTRODUCTION}

Robots have been successfully employed in industrial settings to improve productivity and perform dangerous tasks. In the near future however, due to the recent remarkable improvements in robotic intelligence and technology, it is expected that robots will also coexist with humans to assist or cooperate with them. Therefore, robots must be able to interact with humans in a safe and user-friendly manner while performing cooperative tasks. Joint action requires understanding the other's actions and intentions [1]. To do so, a possible strategy is to transfer knowledge gained from experiments on human-human interaction to technical systems [2].

One basic constituent of robot-human joint action will be physical interaction, e.g., when the human is teaching the robot to assist him in order to solve a complex task. The blueprint for such an interaction is the relation between the master craftsman and his apprentice. In such interactions, a common task is to hand objects from one person (the apprentice) to the other (the master). In the present work we investigated whether current robot technology allows for intuitive and natural joint-action in a hand-over task which simply consisted of 6 wooden cubes being handed over from the apprentice (either human or robot) to the master (human).

Single-handed human multi-joint movements for pointing or reaching are well-studied and various mathematical models have been proposed to describe their kinematics (for review see [3]) based on some optimization criterion, e.g. minimum-jerk [4] or minimum-variance [5]. Studies of the kinematics of grasping similarly revealed characteristic patterns of behavior [6], [7], [8]. Some of these results have already been implemented in robot environments to simulate human behavior [9]. However, studies about cooperative strategies and behavior in humans, specifically concerning manual joint-action, are relatively new. There exist only few examinations about different action pattern for competitive and cooperative behaviors [10], the transfer of objects in joint-action [11], or cooperative lifting of objects [12]. Extending these results to the field of robot-human cooperation raises new questions about acceptance and efficiency. Latest results in this field of research is reported in [13], [14].

In the present work we focus on the comparison of timing in a hand-over task between two humans or a robot and a human. Even though the overall task of receiving a fixed number of objects by hand-over is known to the test subject, many parameters of the exact execution are not specified by the instruction. These parameters, such as position of the hand-over or timing of the movements, depend on the giving subject and become evident by observation during the first few hand-over actions. We assumed that the human receiver flexibly adapts to these parameters and interaction becomes smoother and more rapid within a few repetitions. In the present work, we examined our assumption by tracking hand movements during a human-human handover task. Additionally, we show that basic principles known from human motor control such as smooth velocity profiles and accuracy-dependent velocity scaling of arm movements can also be observed during cooperative action. We then compared human-human to human-robot joint action using the same basic task. In the human-robot experiment, we used two different velocity profiles to drive the end-effector of a humanoid robot. The average movement duration was adapted to that of the previous human-human experiment.

\section{EXPERIMENTAL SETUP}

\section{A. Human-Human Hand-Over Experiment}

We measured hand movements in human subjects during a hand-over task using the magnet-field based motion tracking system Polhemus Liberty. The two test subjects were sitting opposite to each other at a table (width $100 \mathrm{~cm}$ ). The hand positions of the subjects were recorded by the tracking system. Tracking sensors were placed on the back 


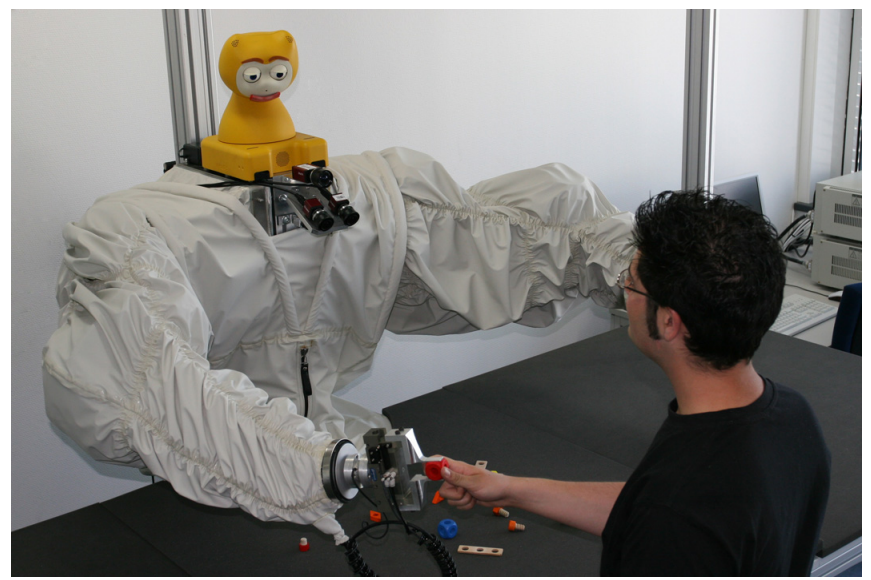

Fig. 1. Setup (JAST project) with two robot arms and an animatronic head.

of the subject's hands and shoulders. Six wooden cubes $(3 \times 3 \times 3 \mathrm{~cm})$ were handed over by one subject (giving subject) to the other (taking subject). The size of the cubes required a precision grip. The cubes were placed in one row on pre-defined marks at the table. The same marks at the other side of the table served as targets for placing the cubes after each hand-over. The distance between the two rows of marks was $80 \mathrm{~cm} .24$ test subjects distributed in 12 pairs (all students and university personal with body heights of 1.60 to $1.90 \mathrm{~m}$ ) participated in the experiment. The instruction to the subjects was given before the experiment started: "The person with the cubes will hand over the cubes, one after the other. The other person should place them on the marks in front of him."

\section{B. Robot-Human Hand-Over Experiment}

In our second experiment, the giving subject was replaced by a robot system (Fig. 11) [15]. The cubes were placed on the table in a straight line and handed over by the robot to 12 test subjects (students). The subjects were again instructed to put each cube on the table after receiving it from the robot. Between the handovers, the subjects should place their hand on the table. The robot was programmed to wait between zero and four seconds between each hand-over, so that the human would not be able to adapt to a periodical behavior. After each trial, the robot readopted its initial resting position in mid-air, while the human subject always started with the hand placed on the table. The hand-over position adopted by the robot was fixed. Two conditions were tested: the hand trajectory of the robot was determined either as minimumjerk trajectory in spatial coordinates or as trapezoidal velocity profile in joint coordinates. The same 12 subjects as above participated in this experiment. The order of conditions was balanced between subjects. For tracking the hand of the human subject, we used the acoustic-based tracking device Intersense IS-600.

In the first condition, we used the minimum jerk model [4] in Cartesian coordinates. This leads to the objective function $\mathrm{c}(\mathrm{r})$ (1), where $\mathbf{r}$ is the grippers positions-vector and $t_{e}$ is the duration of the movement.

$$
c(\mathbf{r})=\frac{1}{2} \int_{0}^{t_{e}}\left|\frac{d^{3} \mathbf{r}}{d t^{3}}\right|^{2} d t
$$

Minimizing this objective function leads to a fifth-order polynomial. Given initial/end position, velocity and acceleration for the trajectory, we can specify the polynomial coefficients. The derivation of this equation results in the velocity profile (2), where $\mathbf{r}_{0}$ and $\mathbf{r}_{e}$ denote the initial and end-positions of the gripper, with the desired duration $t_{e}$.

$$
\dot{\mathbf{r}}(t)=\left(\mathbf{r}_{0}-\mathbf{r}_{e}\right)\left(60 \frac{t^{3}}{t_{e}^{4}}-30 \frac{t^{4}}{t_{e}^{5}}-30 \frac{t^{2}}{t_{e}^{3}}\right)
$$

A recorded velocity profile for a single movement is shown in Fig. 2 (left upper corner). The corresponding trajectories in Cartesian space for all 6 cube positions are plotted in Fig. 2 (right upper corner). Interpolation was performed in Cartesian space of the robot, resulting in straight lines.

The second set of trajectories was calculated based on a trapezoidal velocity profile in joint coordinates $\dot{\theta}(t)$, with a constant acceleration $\ddot{\theta}_{a}$ and deceleration $\ddot{\theta}_{d}$ phase (4). $t_{a}$ stands for the acceleration, $t_{d}$ for the deceleration time. Because of the joint coordinates, the trajectories are not straight like in the minimum jerk profile (Fig. 2, lower right corner). The recorded velocity profile does not show a trapezoidal shape because of the transition from joint coordinates to Cartesian coordinates (Fig. 2, lower left corner)).

$$
\begin{gathered}
\dot{\theta}(t)= \begin{cases}\ddot{\theta}_{a} t+\dot{\theta}_{0}, & 0 \leq t<t_{a} \\
\ddot{\theta}_{a} t_{a}+\dot{\theta}_{0}, & t_{a} \leq t<t_{d} \\
\ddot{\theta}_{a} t_{a}+\ddot{\theta}_{d}\left(t-t_{d}\right)+\dot{\theta}_{0}, & t_{d} \leq t<t_{e}\end{cases} \\
\ddot{\theta}(t)= \begin{cases}\ddot{\theta}_{a}, \quad 0 \leq t<t_{a} \\
0, & t_{a} \leq t<t_{d} \\
\ddot{\theta}_{d}, & t_{d} \leq t<t_{e}\end{cases}
\end{gathered}
$$

Instead of calculating the trajectory off-line before each movement, an on-line calculation after each update step of the robot controller is possible. In the present experiment however, we only included a joint-space interpolation for the trajectory, resulting in curves in the Cartesian space. Because of the discrete acceleration function (4), there is an overshoot for the velocity in the mechanical system.

The parameters for the velocity profiles were adapted in order for the robot to take about $1.2 \mathrm{~s}$ for each point to point trajectory. This movement duration was taken from the trajectories recorded in the human-human experiments. The maximum velocities of the robot were calculated from the duration parameter. After the experiment, the subjects were asked questions about how human-like the movements were and how secure they felt. Each question had to be scored from 1 (not at all) to 5 (very much).

\section{RESUlts}

\section{A. Human-Human Hand-Over Experiment}

A typical set of human-human data is shown in Fig. 3. with the z-coordinate (height over table) of the hands of two 

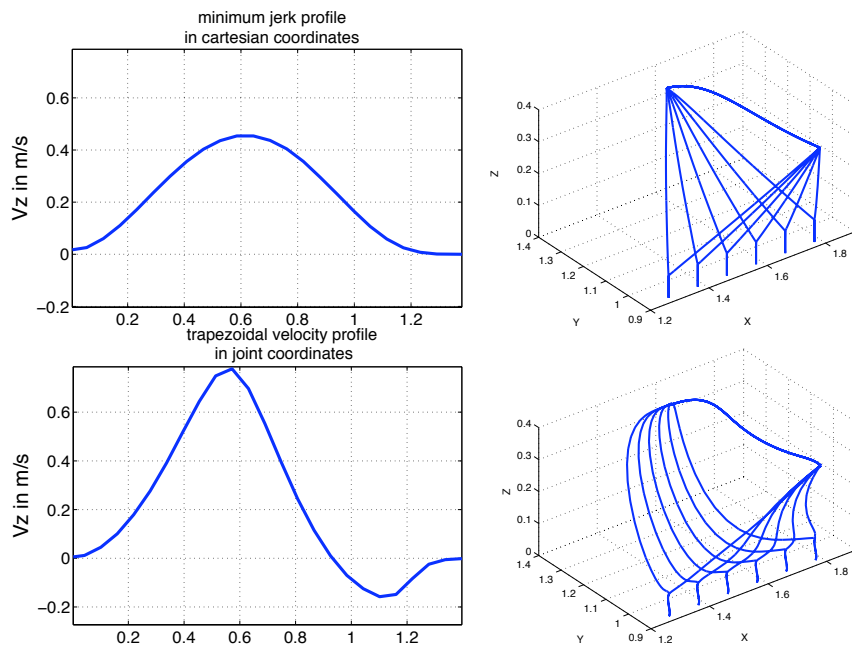

Fig. 2. Left: examples of the robot velocity over time for each condition (top: minimum jerk, bottom: trapezoidal). Right: corresponding trajectories of the robot gripper in Cartesian space for the different six cube positions. The pre-defined hand-over position is located in the upper left part, the initial waiting position in the upper right part. The robot gripper moves from the initial waiting position to one of the cube positions (bottom positions) and from there to the hand-over position.

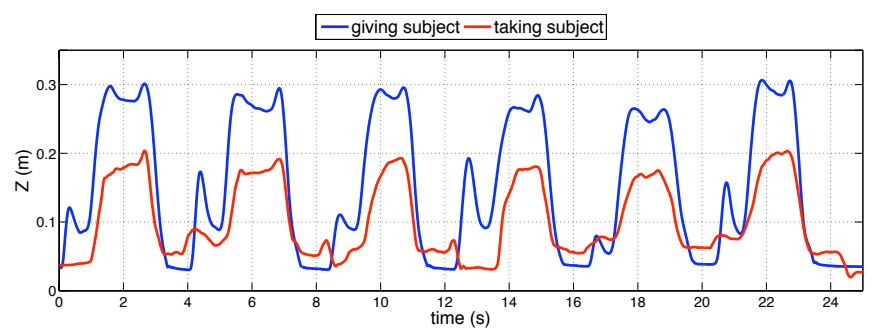

Fig. 3. Typical trajectory of hand movements during the hand-over task The height of the hand over the table is plotted over the time. Blue: giving subject, red: taking subject.

human subjects over time. In this example, the six hand-over maneuvers took about $25 \mathrm{~s}$. The giving subject (blue trace) first lifted his hand to reach out and grab the first cube (small peak), then moved it to the hand-over position. The taking subject (red) reacted and started movement towards the handover position, while the hand of the giving subject was still in motion. After the transfer of the object, both subjects descended their hands, and the taking subject's hand shows an additionally short peak corresponding to cube placement on the table. Movements were smooth with typical bellshaped velocity profiles. The mean peak velocities of the subjects were $0.93 \mathrm{~m} / \mathrm{s}( \pm 0.19)$ for the giving and $0.85 \mathrm{~m} / \mathrm{s}$ $( \pm 0.12)$ for the taking subject. Peak velocity was higher without the object than with the object.

For the analysis of hand-over timing we defined three time-sections (s. Fig. 4). The reaction-time was defined as the duration between the velocity peaks of the giving and taking subjects. Manipulation-time is the duration between velocity peaks for ascending and descending movements by the taking subject. A third time-section, called posthandover-time was defined as duration between the velocity

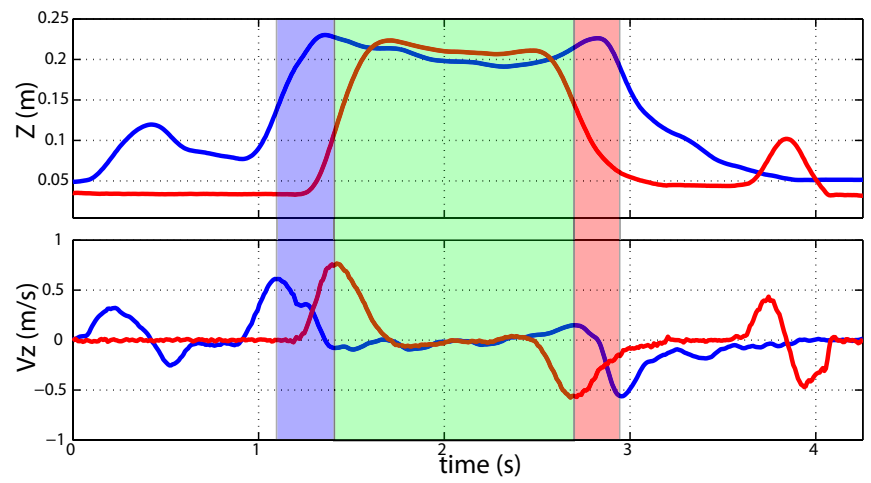

Fig. 4. Definition of time-sections for analysis: reaction time (blue), manipulation time (green), and post-handover-time (orange).

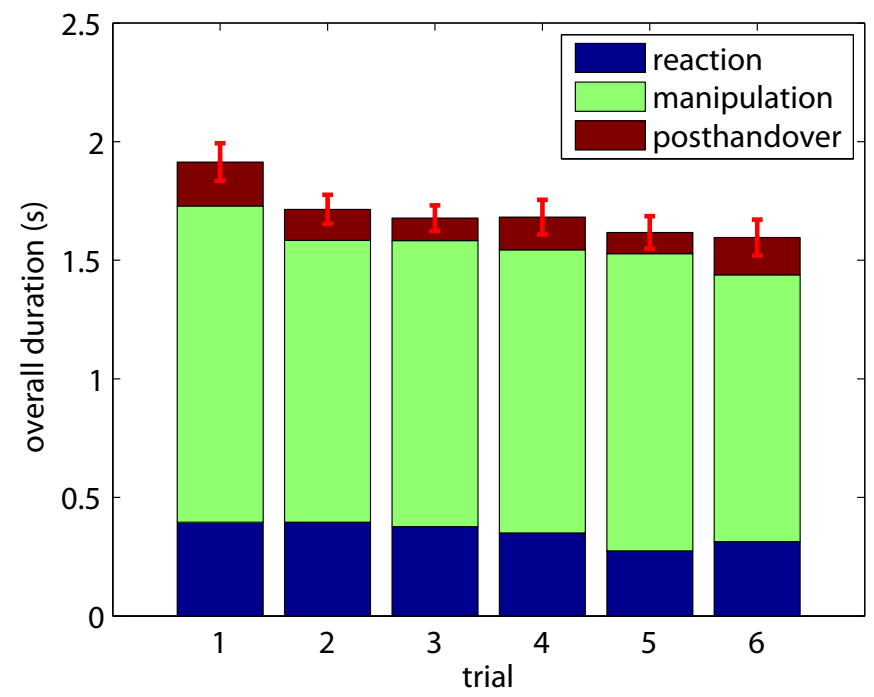

Fig. 5. Overall hand-over duration for all six trials together with the respective time sections (blue: reaction time, green: manipulation time, red: post-handover-time). Error bars indicate SEM of overall duration.

peaks of the descending movements of both subjects. The sum of those durations defines overall hand-over duration.

Repeated measures (ANOVA) showed significant effect of trial number for overall duration $[\mathrm{F}(5,55)=5.34 ; \mathrm{p}=0.0004]$ due to a decrease in duration with increasing number of trials. However, this effect could not be attributed to specific movement sections (Fig. 5), but consisted in a combined reduction of reaction and post-handover times, while manipulation time stayed roughly constant over repetitions.

Table II shows average duration of each time section together with the corresponding standard deviation. A closer inspection of the reaction time data showed that in the last trial some taking subjects often even anticipated movement, resulting in an increased variability of reaction time ( $\mathrm{SD}=0.33$ compared to an average of $\mathrm{SD}=0.25$ in the first five trials ). Thus, the trend towards a decrease in reaction time over trial only became significant when analysis was restricted to the first 5 hand-overs $[\mathrm{F}(4,44)=2.67 ; \mathrm{p}=0.044]$.

As can be seen in Table $\mathbb{1}$ the standard deviations of single time sections are often larger than that of their sum (overall duration). This means that single time sections are not 
TABLE I

AVERAGE DURATION AND STANDARD DEVIATION OF THE TIME SECTIONS OF EACH HAND-OVER TRIAL IN SECONDS.

\begin{tabular}{c||c|c|c|c|c|c}
\hline Time section & 1 & 2 & 3 & 4 & 5 & 6 \\
\hline \hline Reaction time & & & & & & \\
mean & 0.40 & 0.40 & 0.38 & 0.35 & 0.27 & 0.31 \\
SD & 0.19 & 0.31 & 0.25 & 0.25 & 0.26 & 0.33 \\
\hline $\begin{array}{c}\text { Manipulation time } \\
\text { mean }\end{array}$ & 1.33 & 1.18 & 1.20 & 1.19 & 1.25 & 1.13 \\
SD & 0.24 & 0.33 & 0.37 & 0.37 & 0.41 & 0.36 \\
\hline Post-handover time & & & & & & \\
mean & 0.18 & 0.13 & 0.10 & 0.14 & 0.09 & 0.16 \\
SD & 0.21 & 0.18 & 0.34 & 0.19 & 0.21 & 0.26 \\
\hline Overall duration & & & & & & \\
mean & 1.91 & 1.71 & 1.68 & 1.68 & 1.62 & 1.60 \\
SD & 0.27 & 0.21 & 0.19 & 0.25 & 0.23 & 0.26 \\
\hline
\end{tabular}
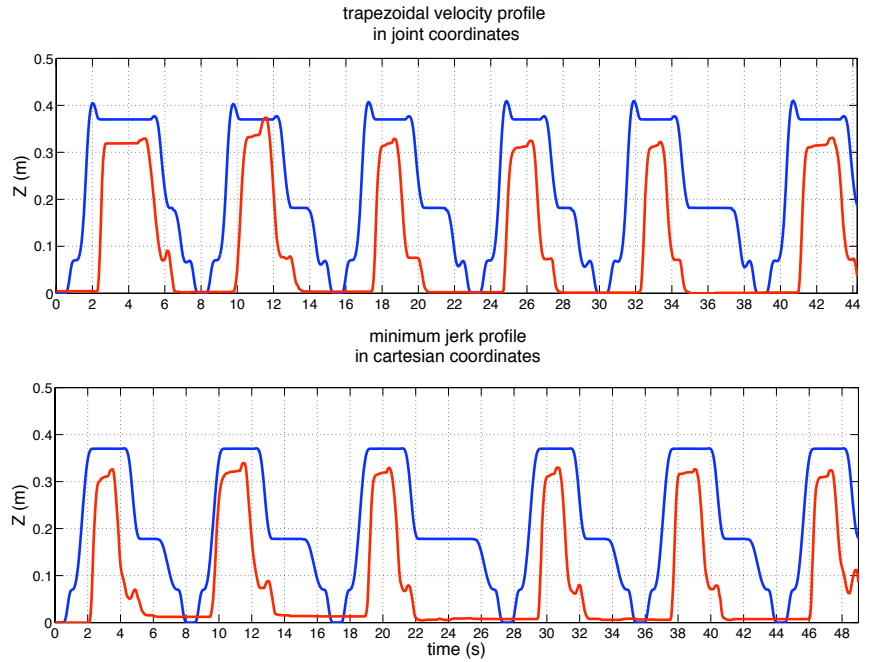

Fig. 6. Typical trajectory of hand and gripper movements during the handover task. The height of the hand (red) and the gripper (blue) over the table is plotted over the time.

statistically independent. The correlation coefficient between the reaction time and the manipulation time is calculated to be $\mathrm{r}=-0.67, \mathrm{p}<0.001$, the correlation for manipulation time and post-handover time is calculated to be $\mathrm{r}=-0.49, \mathrm{p}<0.001$. Thus, on average, longer reaction time led to shorter manipulation time and vice versa. We assume that the relative gain in duration caused by a rapid or even anticipatory reaction is often cancelled by requiring subsequent fine tuning of grasping posture or exact handover position.

\section{B. Robot-Human Hand-Over Experiment}

In the second experiment, the giving subject was replaced by the robot. Two different velocity profiles were installed in the robot. In Fig. 6, the height of the hand (red) and the gripper (blue) above the table over time is plotted. The human trajectory showed the same behavior as in our humanhuman experiment. The robot trajectory started from its initial position (approx. $0.18 \mathrm{~m}$ above the table). Subsequently, the gripper went down to grasp the cube and lift it to the pre-defined hand-over position. After the hand-over the robot arm moved back to its initial position.
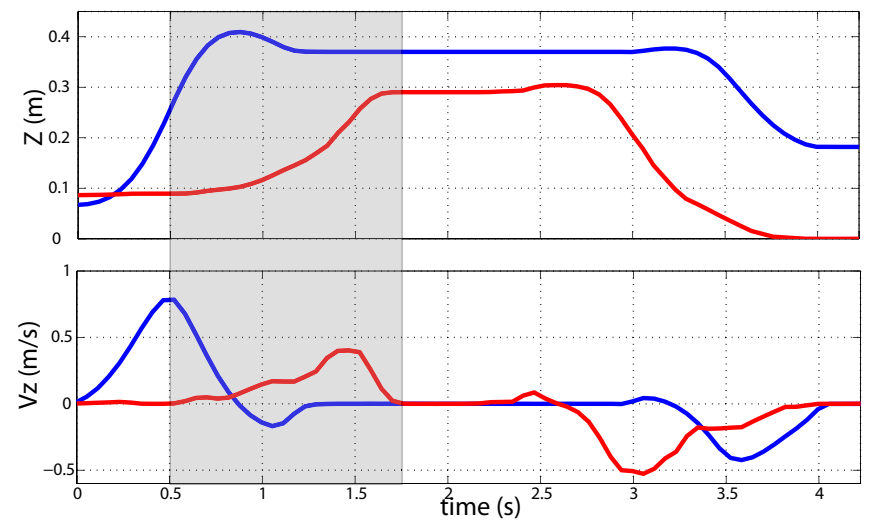

Fig. 7. Plot of a handover trajectory from one of the subjects (red curve) with a non bell-shaped velocity profile (grey part of the trajectory).

Three subjects in both conditions showed a different behavior than the others. These subjects showed an online correction of their hand velocity during at least one hand-over. The respective velocity profiles showed a short delay with subsequent deceleration followed by a second acceleration to reach the handover position. This behavior resulted in a non bell-shaped velocity profile (Fig. 7). Since comparison of reaction times of these profiles with the usual bell-shaped profiles are not feasible on the basis of peak velocity, data from these subjects was not taken into account for the statistical analysis. One other subject was removed from the analysis due to sensor artifacts.

Repeated measures (ANOVA) for the overall duration of a single hand-over showed a significant main effect of condition $[\mathrm{F}(1,7)=7.55 ; \mathrm{p}=0.029]$ with shorter overall time for the minimum-jerk profiles $(2.68 \pm 0.08 \mathrm{~s}$ vs. $2.96 \pm 0.14 \mathrm{~s}$ mean \pm SEM), but no effect for trial number or interaction. However, this effect was mainly due to an artifact: in the trapezoidal condition, gripper sensors for object release had to be switched on about $0.5 \mathrm{~s}$ later in order to avoid dropping the object due to forces generated by the oscillation of the robot arm, thus increasing the manipulation time.

The reaction times of the taking subject for both conditions are shown in Fig. 8 Statistical analysis revealed that reaction times in the minimum jerk condition $(0.39 \pm 0.04 \mathrm{~s})$ were significantly shorter $[\mathrm{F}(1,7)=9.74 ; \mathrm{p}=0.017]$ than in the trapezoidal condition $(0.50 \pm 0.06 \mathrm{~s})$. Additionally, a significant interaction between condition and trial number $[\mathrm{F}(5,35)=3.47 ; \mathrm{p}=0.012]$ confirmed the difference in trial-totrial evolution seen in Fig. 8. reaction times decreased for minimum jerk, but not for trapezoidal velocity. This is most notable in the first three trials, where the influence of the unpredictable inter-trial duration can be neglected.

The post-experimental questionnaire filled out by the test subjects ( statistics shown in Fig. 9) revealed that the subjects did not recognize any difference between the two profiles in terms of human-like motion. However, subjective safety was significantly larger for the the minimum jerk profile (Wilcoxon matched pairs test, $\mathrm{p}=0.013$ ).

The average peak velocity of the various trajectories of the 


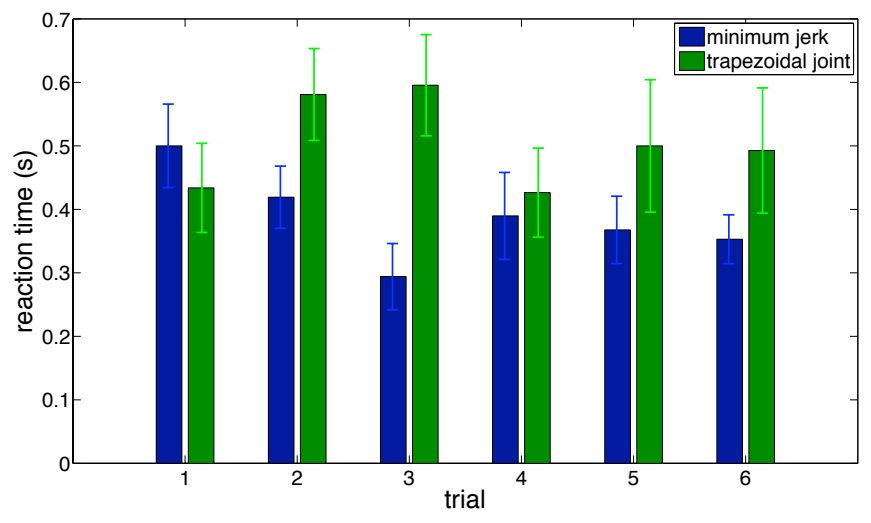

Fig. 8. Reaction time for the six handovers between the robot and the human. Blue bars show the reaction times for the minimum jerk velocity profile, green bars for the trapezoid velocity profile. Error bars show standard deviation.
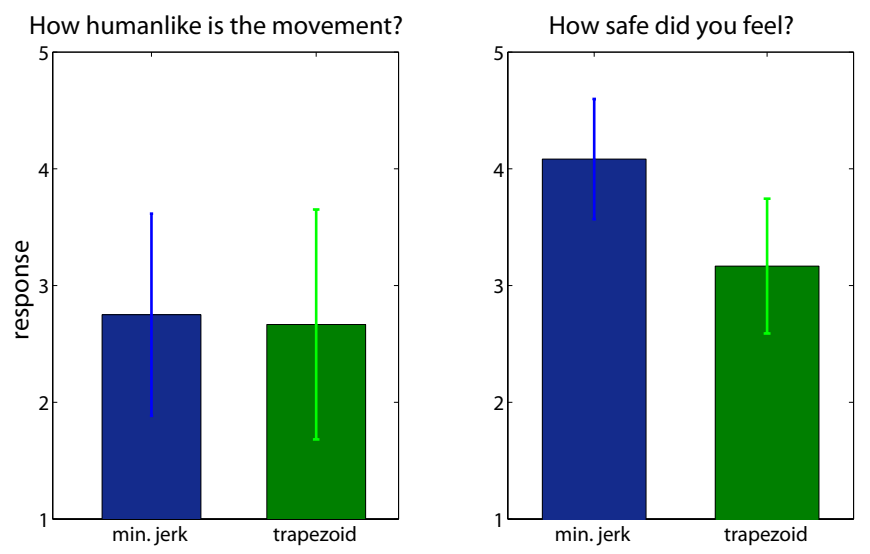

Fig. 9. Interview of the subjects after the experiments: the subjects had to answer (from 1 to 5) how human-like they thought the robot movement was and how safe they felt during the experiment.

robot was around $0.97 \mathrm{~m} / \mathrm{s}$ for minimum jerk in Cartesian space and $1.46 \mathrm{~m} / \mathrm{s}$ for the trapezoidal velocity profile. The average peak velocity of the subjects was at about $0.85 \mathrm{~m} / \mathrm{s}$ which corresponds with the velocities measured in the human-human experiment.

\section{Comparison of Human-Human and Robot-Human Hand- Over Experiments}

In order to compare the results of the two experiments, we chose to only consider the robot-human condition using minimum jerk trajectories. A mixed ANOVA for overall duration of handover revealed a significant difference between humanhuman and human-robot hand-over $[\mathrm{F}(1,19)=124$; $\mathrm{p}<0.001]$ due to average duration being almost $1 \mathrm{~s}$ longer for robothuman hand-over. An effect of trial $[\mathrm{F}(5,95)=3.77 ; \mathrm{p}=0.004]$ without significant interaction confirmed that overall duration decreased in both conditions over time. Manipulation time showed a small but significant effect of condition $[\mathrm{F}(1,19)=5.54 ; \mathrm{p}=0.03]$ with slightly larger duration in the robot-human task $(1.49 \pm 0.09 \mathrm{~s}$ vs. $1.22 \pm 0.08 \mathrm{~s}$ for humanhuman). Analysis of reaction time revealed a significant effect of trial number $[\mathrm{F}(5,95)=2.48 ; \mathrm{p}=0.037]$ due to the de-

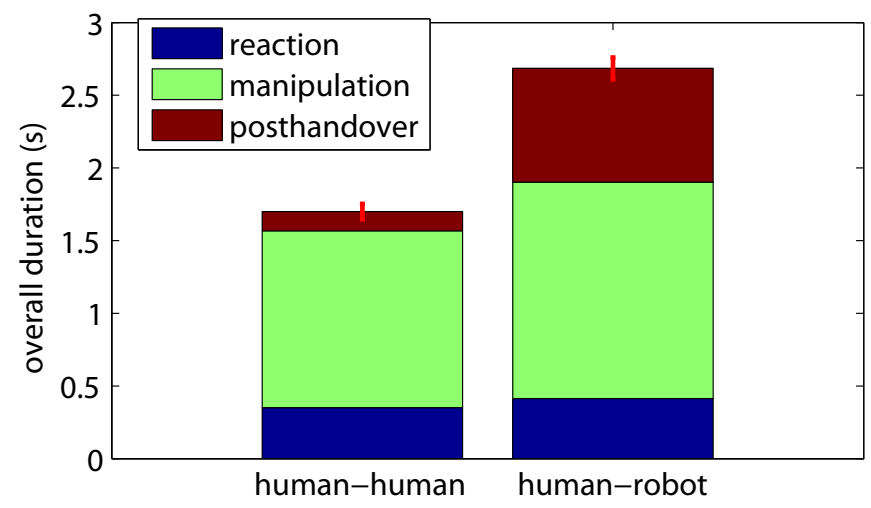

Fig. 10. Comparison of hand-over durations for human-human and humanrobot joint action. Error bars show standard error of the mean.

crease in reaction time for increasing trial number. However, no significant difference between human-human or robothuman reaction time was found.

Thus, surprisingly, the main reason for the difference in overall duration (Fig. 10) was not reaction time or manipulation time, but post-handover duration (humanhuman: $0.13 \pm 0.05 \mathrm{~s}$; robot-human: $0.78 \pm 0.05 \mathrm{~s}$ ). Since posthandover time is determined by the giving subject, the reason for this finding becomes clear: the robot did not retract his gripper as fast as the human his hand in the same situation.

\section{Discussion}

The present investigation of dyadic manual joint-action is a first attempt to better understand the mechanisms of coordinating sequences of actions both between human subjects and between robots and humans. We could show that, in humanhuman hand-over, the overall duration of a single handover decreased systematically over the six trials studied. In the robot-human hand-over experiments, better results were achieved when using a more natural minimum-jerk velocity profile in spatial coordinates than when performing the action using the conventional trapezoidal velocity profile in joint space. Comparison of human-human and robothuman interaction revealed that the present robot technology, despite apparent disadvantages such as using grippers instead of hands, allows for surprisingly efficient robot-human handover.

While previous related work [11], [12] concentrated on implicit knowledge transfer or synergistic force production, we were interested in the process of achieving smooth and efficient cooperation. The simple task of handing over objects from one human to another, which had not been studied so far, already requires that multiple basic parameters such as the position and timing of the hand-over or the orientation of the object are negotiated during the first actions. This negotiation of interaction boundaries, which then allows fast and smooth cooperation, became evident in the decrease of reaction time and overall duration in our human-human experiment. We could further show that the durations of the different phases of the hand-over are not independent, but influenced by the previous phase. This led to the observed 
smaller variance of overall duration compared to that of single sections of the hand-over action. We assume that, for example, a fast reaction time may lead to an inappropriate hand-position, which then has to be adjusted for an optimal grasp, leading to an increased manipulation time. In general, the hand movements obeyed basic principles of human motor control such as bell-shaped velocity profiles, higher velocity with larger movement amplitude, and slower movements when higher accuracy was required: the hand movement of the receiver, who must reach the object with high accuracy, was slower than that of the giving subject, whose end position was not confined.

The results of the human-human experiment provided a useful baseline for our second experiment, in which the giving subject was replaced by a humanoid robot. In order to evaluate the role of robotic vs. biological motion, we compared two types of velocity profiles. The point to point movement time for both robot-velocity profiles where set to $1.2 \mathrm{~s}$, a duration taken from the human data. For the more biological minimum-jerk velocity profile, average reaction time was significantly shorter and showed a tendency to decrease adaptively over trials, which was not the case for velocity trapezoids in joint space. However, generalizing the minimum jerk profile into a three-dimensional space does not lead to human-like trajectories. As also shown by our own data (not illustrated), human trajectories in space show a curved form usually confined to a plane [16] rather than straight lines. Matching not only the velocity profile but also the trajectory, e.g., by implementing minimum variance profiles [17], may thus lead to even better performance. In turn, unusual behavior such as the on-line corrections performed by three of our subjects, may be avoided. The significantly higher manipulation time with trapezoidal profiles was due to an artificially longer gripper closure to avoid object slippage. Notably, this small difference in the protocol became directly visible as significant prolongation of the recorded manipulation time, suggesting that human manipulation is extremely efficient.

In contrast to a previous report [18] showing that human subjects considered the maximum velocity of a planar handing-over machine as acceptable at about $0.225 \mathrm{~m} / \mathrm{s}$, our subjects considered the chosen peak robot velocity of about $1 \mathrm{~m} / \mathrm{s}$ as subjectively safe. More importantly, the timing of the human hand movements in this task showed that subjects indeed felt confident about physically interacting with the robot: reaction times in human-human and robot-human interaction were not significantly different, which clearly demonstrates that the robot movement was as predictable as that of a human.

Further comparison with the human-human scenario showed that robot-human hand-over duration was approximately $1 \mathrm{~s}$ longer. This prolonged duration was mainly due to the last section of the hand-over, in which our robot was programmed to wait significantly longer than the average human to retract his gripper towards the start position. Moreover, trial-to-trial adaptation of overall duration was not significantly different between human-human and robot-human scenarios. In summary, our present comparison shows that robot-human hand-over as performed in our setup almost matches the speed and efficiency of humanhuman hand-over. Properties such as trial-to-trial adaptation or anticipatory reach-to-grasp movements were equally seen in both scenarios. Nonetheless, optimizing other parts of the action sequence not considered here such as the gripping of the object will be necessary to achieve the same overall efficiency as in human-human hand-over.

\section{ACKNOWLEDGEMENT}

This work is supported by the DFG excellence initiative research cluster "CoTeSys" (www. cotesys.org) and by the EU FP6 IST Cognitive Systems Integrated Project "JAST" (FP6-003747-IP) (wWw. euprojects-jast.net).

\section{REFERENCES}

[1] U. Castiello, "Understanding other people's actions: Intention and attention," Journal of Experimental Psychology: Human Perception and Performance, vol. 29, no. 2, pp. 416-430, 2003.

[2] W. Erlhagen, A. Mukovskiy, E. Bicho, G. Panin, C. Kiss, A. Knoll, H. van Schie, and H. Bekkering, "Goal-directed imitation for robots: A bio-inspired approach to action understanding and skill learning," Robotics and Autonomous Systems, vol. 54, no. 5, pp. 353-360, 2006.

[3] S. E. Engelbrecht, "Minimum principles in motor control," Journal of Mathematical Psychology, vol. 45, no. 3, pp. 497-542, 2001.

[4] T. Flash and N. Hogan, "The coordination of arm movements: An experimentally confirmed mathematical-model," Journal of Neuroscience, vol. 5, no. 7, pp. 1688-1703, 1985.

[5] C. M. Harris and D. M. Wolpert, "Signal-dependent noise determines motor planning," Nature, vol. 394, no. 6695, pp. 780-784, 1998.

[6] M. Jeannerod, "Intersegmental coordination during reaching at natural visual objects," in Attention and Performance IX. Lawrence Erlbaum, 1981, pp. 153-169.

[7] - "The timing of natural prehension movements," Journal of Motor Behavior, pp. 235-254, 1984.

[8] J. B. J. Smeets and E. Brenner, "A new view on grasping," Motor Control, vol. 3, no. 3, pp. 237-271, 1999.

[9] G. Simmons and Y. Demiris, "Object grasping using the minimum variance model," Biological Cybernetics, vol. 94, no. 5, pp. 393-407, 2006.

[10] I. Georgiou, C. Becchio, S. Glover, and U. Castiello, "Different action patterns for cooperative and competitive behaviour," Cognition, vol. 102, no. 3, pp. 415-433, 2007.

[11] R. G. J. Meulenbroek, J. Bosga, M. Hulstijn, and S. Miedl, "Jointaction coordination in transferring objects," Experimental Brain Research, vol. 180, no. 2, pp. 333-343, 2007.

[12] J. Bosga and R. G. J. Meulenbroek, "Joint-action coordination of redundant force contributions in a virtual lifting task," Motor Control, vol. 11, no. 3, pp. 235-258, 2007.

[13] S. Shibata, T. Yamamoto, M. Jindai, and A. Shimizu, "A fundamental approach of avoidance planning of robots considering human emotions," JSME International Journal Series C, vol. 46, no. 1, pp. 270277, 2003.

[14] S. Shibata and H. Inooka, "Psychological evaluations of robot motions," International Journal of Industrial Ergonomics, vol. 21, no. 6, pp. 483-494, 1998.

[15] M. Rickert, M. E. Foster, M. Giuliani, T. By, G. Panin, and A. Knoll, "Integrating language, vision and action for human robot dialog systems," in Proceedings of the International Conference on HumanComputer Interaction, 2007, pp. 987-995.

[16] M. D. K. Breteler, R. G. J. Meulenbroek, and S. C. A. M. Gielen, "Geometric features of workspace and joint-space paths of $3 \mathrm{~d}$ reaching movements," Acta Psychologica, vol. 100, no. 1-2, pp. 37-53, 1998.

[17] G. Simmons and Y. Demiris, "Optimal robot arm control using the minimum variance model," Journal of Robotic Systems, vol. 22, no. 11, pp. 677-690, 2005.

[18] M. Jindai, S. Shibata, T. Yamamoto, and A. Shimizu, "A study on robot-human system with consideration of individual preferences," JSME International Journal Series C, vol. 46, no. 3, pp. 1075-1083, 2003. 